Swarthmore College

Works

$10-25-2005$

\title{
Aggregation Behavior And Chromonic Liquid Crystal Properties Of An Anionic Monoazo Dye
}

Viva R. Horowitz , '05

L. A. Janowitz

Aaron L. Modic , '04

P. A. Heiney

Peter J. Collings

Swarthmore College, pcollin1@swarthmore.edu

Follow this and additional works at: https://works.swarthmore.edu/fac-physics

Part of the Physics Commons

Let us know how access to these works benefits you

\section{Recommended Citation}

Viva R. Horowitz , '05; L. A. Janowitz; Aaron L. Modic , '04; P. A. Heiney; and Peter J. Collings. (2005). "Aggregation Behavior And Chromonic Liquid Crystal Properties Of An Anionic Monoazo Dye". Physical Review E. Volume 72, Issue 4. DOI: 10.1103/PhysRevE.72.041710

https://works.swarthmore.edu/fac-physics/132

This work is brought to you for free by Swarthmore College Libraries' Works. It has been accepted for inclusion in Physics \& Astronomy Faculty Works by an authorized administrator of Works. For more information, please contact myworks@swarthmore.edu. 


\title{
Aggregation behavior and chromonic liquid crystal properties of an anionic monoazo dye
}

\author{
Viva R. Horowitz, ${ }^{1}$ Lauren A. Janowitz, ${ }^{1}$ Aaron L. Modic, ${ }^{1}$ Paul A. Heiney, ${ }^{2}$ and Peter J. Collings ${ }^{1,2}$ \\ ${ }^{1}$ Department of Physics \& Astronomy, Swarthmore College, Swarthmore, Pennsylvania 19081, USA \\ ${ }^{2}$ Department of Physics \& Astronomy, University of Pennsylvania, Philadelphia, Pennsylvania 19104, USA
}

(Received 17 March 2005; published 25 October 2005)

\begin{abstract}
$\mathrm{X}$-ray scattering and various optical techniques are utilized to study the aggregation process and chromonic liquid crystal phase of the anionic monoazo dye Sunset Yellow FCF. The x-ray results demonstrate that aggregation involves $\pi$ - $\pi$ stacking of the molecules into columns, with the columns undergoing a phase transition to an orientationally ordered chromonic liquid crystal phase at high dye concentration. Optical absorption measurements on dilute solutions reveal that the aggregation takes place at all concentrations, with the average aggregation number increasing with concentration. A simple theory based on the law of mass action and an isodesmic aggregation process is in excellent agreement with the experimental data and yields a value for the "bond" energy between molecules in an aggregate. Measurements of the birefringence and order parameter are also performed as a function of temperature in the chromonic liquid crystal phase. The agreement between these results and a more complicated theory of aggregation is quite reasonable. Overall, these results both confirm that the aggregation process for some dyes is isodesmic and provide a second example of a well-characterized chromonic system.
\end{abstract}

DOI: 10.1103/PhysRevE.72.041710

PACS number(s): 61.30.-v, 61.10.Eq, 61.25.Hq, 42.70.Df

\section{INTRODUCTION}

Chromonic liquid crystals represent an intersection of two very active fields of research, supramolecular assembly and ordered complex fluids. On the one hand, the spontaneous aggregation of some molecules due to a combination of weak attractive forces and solvent effects is a rich field of current scientific inquiry, with possible applications ranging from the generation of new materials to the treatment of disease. On the other hand, liquid crystals represent a class of complex fluids that has revealed a great deal about molecular interactions, phase transitions, and the science of soft condensed matter in general. In the chromonic liquid crystal phase, an aqueous solution of a dye, drug, or nucleic acid assembles to form aggregates which are anisotropic in shape (rodlike or disklike). If the concentration of these aggregates is high enough and the shape of these aggregates is anisotropic enough, a nematic liquid crystal phase forms (the chromonic $N$ phase) in which the aggregate axes possess a preferred direction as the aggregates diffuse, break up, and reform. At even higher concentration and/or lower temperature, a second liquid crystal phase sometimes forms in which the aggregates are also positionally ordered in a hexagonal array (the chromonic $M$ phase). Chromonic liquid crystals are lyotropic liquid crystals, but in general they do not possess a critical micelle concentration as is normally the case for the lyotropic liquid crystals formed by amphiphilic molecules. Rather, the process of aggregation in chromonic systems has been described as isodesmic, meaning that the "bond" energy between molecules in an aggregate is independent of aggregate size.

The existence of chromonic liquid crystals has been known for some time. Many of the studies have focused on disodium cromoglycate, a drug developed for the treatment of asthma, which forms a liquid crystal phase at room temperature for concentrations higher than about $10 \mathrm{wt} \%[1-3]$. $\mathrm{X}$-ray measurements reveal the existence of both chromonic
$N$ and $M$ phases with a repeat distance of $0.34 \mathrm{~nm}$ between the aromatic rings of neighboring molecules in the aggregate and a spacing between the centers of $2-3 \mathrm{~nm}$ diameter columns of about $4 \mathrm{~nm}[4,5]$. Nuclear magnetic resonance studies show a very high value for the order parameter in these phases [5]. Polarized infrared spectroscopy experiments yield lower values of the order parameter for various bonds in the molecule, which is not surprising because the bonds are not aligned with respect to the aggregate axis [6]. Very recently, a light scattering and viscosity study concluded that the diameter of the columns is about $2 \mathrm{~nm}$ (about the size of a single molecule) and the average length of the columns at the isotropic - nematic phase transition is about $20 \mathrm{~nm}$ [7]. The structure of the aggregates is still not known with certainty, with some measurements suggesting a column of single molecules [4], and with some results favoring a structure with four molecules in the cross section of a hollow column $[6,8,9]$. The birefringence of the nematic phase is negative and small, which is consistent with the formation of columnar aggregates in which the molecular axis with the largest index of refraction is perpendicular to the long axis of the column $[4,10,11]$.

Investigations of other chromonic liquid crystal systems are not nearly as extensive as those on disodium cromoglycate. There have been many studies of pseudoisocyanine chloride solutions, but few of the results are for concentrated samples where the liquid crystal phase appears [12-14]. Other work has investigated a number of dyes including xanthone derivatives [15], benzopurpurin 4B [16], acid red 266 [17], phthalocyanine and porphyrin derivatives [18], Levafix Goldgelb [19], Violet 20 [11,20], direct blue 67 [21,22], and Blue 27 [11]. These studies suggest a number of general conclusions for dye systems. First, a liquid crystal phase normally appears at room temperature for concentrations in the 5-15 wt \% range. Second, evidence points to both chromonic $N$ and $M$ phases in most of these systems. Third, an x-ray scattering peak corresponding to a $0.34 \mathrm{~nm}$ repeat distance is 
usually present. Fourth, there is evidence that aggregation takes place even at low concentrations of the dye, with the aggregates increasing in size as the concentration increases. Fifth, while suggestions exist as to the structure of the aggregates in these systems, the evidence favoring one particular structure is even more limited than for disodium cromoglycate.

In order to understand the behavior of chromonic liquid crystals, it is important to study both the aggregation process and the properties of the liquid crystal phases. To complement the extensive work on disodium cromoglycate, a different system was selected, one with a simpler molecular structure which is representative of the many dyes known to aggregate and form liquid crystals. The anionic monoazo food dye Sunset Yellow FCF (C. I. Food Yellow 3 or FD \& C Yellow 6), the disodium salt of 6-hydroxy-5[(4-sulfophenyl)azo]-2-naphthalenesulfonic acid, has been reported to form a liquid crystal phase in one unpublished report [23] and was investigated using optical, magnetic, and $\mathrm{x}$-ray techniques in another unpublished report by Luoma [24]. Because Sunset Yellow FCF is a food coloring, it is available in a purer form than many textile dyes (as is also the case for the drug disodium cromoglycate). From this previous work, it is clear that Sunset Yellow FCF forms a liquid crystal phase at room temperature for concentrations greater than about $25 \mathrm{wt} \%$ and that the repeat distance is again 0.34 $\mathrm{nm}$. Luoma also provides evidence that (1) the aggregation starts at low concentrations with the formation of small aggregates and continues with the formation of larger aggregates as the concentration gets higher, (2) in the liquid crystal phase aggregates contain on the order of ten molecules, (3) the length of the aggregate varies with the square root of the volume fraction, (4) the diameter of the aggregates is about $1.4 \mathrm{~nm}$, and (5) the order parameter of the aggregates varies between 0.75 and 0.9 . In an effort to investigate the aggregation process and liquid crystal properties of this promising system further, uv-visible absorption spectra and x-ray scattering profiles were obtained on solutions too dilute for the liquid crystal phase to form. Following Luoma's investigation [24], the diffuse $\mathrm{x}$-ray peak due to aggregate-aggregate distances was analyzed, but this time over a much wider concentration range. In addition, fundamental measurements on the nematic phase of Sunset Yellow FCF were performed, revealing how the birefringence depends on temperature and concentration and what the order parameter variation with temperature is for a typical concentration. Finally, a very simple theory of aggregation was tested against the absorption data and the agreement is excellent. A more complicated theory of aggregation, which should be valid in the liquid crystal phase, is in reasonable agreement with the experimental results for more concentrated solutions. Thus the work reported here represents a comprehensive study of a chromonic liquid crystal other than disodium cromoglycate. By performing different measurements over a concentration range that includes both the isotropic and liquid crystal phase, this investigation is in some senses more complete than most previous studies. But most interesting, perhaps, are the large number of similarities between the two systems. Undoubtedly, some systems behave quite differently, but at this point the basic behavior of a class of chromonic liquid crystals is becoming clear.

\section{THEORETICAL CONSIDERATIONS}

There has not been a great deal of theoretical work on chromonic liquid crystals. If the aggregates are considered to be uniform rods or spherocylinders that interact through the excluded volume effect, then the calculations of Onsager [25] and Lee [26], respectively, can be applied. The former is only applicable in the limit of very long rods. The latter predicts a volume fraction change of about $8 \%$ and an orientational order parameter of about 0.7 at the nematic-isotropic transition for a volume fraction of about 0.28 and a length to diameter ratio of 9. Taylor and Herzfeld consider selfassembling systems of linear aggregates, including in the calculation hard-core potentials, short-range repulsions, and an energy contribution for each pair of molecules [27,28]. The distribution of aggregate sizes depends on the parameters controlling the interactions, but a typical system with a volume fraction of about 0.28 has about six molecules in an aggregate on average and an orientational order parameter of 0.7 at the transition. Recently a Monte Carlo simulation of a mixture of model chromonic and water molecules has been performed [29]. Two different models for the chromonic molecules are employed and both attractive and repulsive interactions are introduced among the units comprising the model of the chromonic molecule and the model of the water molecule. The simulation shows a gradual increase in the average number of molecules per aggregate, reaching about ten at a volume fraction of about 0.1. Using one model for the chromonic molecule yields columnar aggregates while using another model for the chromonic molecule does not. At high concentrations, the columnar aggregates become more chainlike.

On the other hand, very simple theoretical ideas have been used with some success on systems that spontaneously self-assemble. For example, Israelachvili examines the behavior of a system of aggregates by invoking the law of mass action to describe the equilibrium between $N$ individual molecules and an aggregate of $N$ molecules [30]. If $X_{N}$ is the volume fraction of molecules in aggregates of size $N$, and if $\mu_{N}^{0}$ is the mean interaction free energy per molecule in an aggregate of $N$ molecules, then $X_{N}$ can be written in terms of the volume fraction of the individual molecules $X_{1}$ :

$$
X_{N}=N\left(X_{1} e^{-\left(\mu_{N}^{0}-\mu_{1}^{0}\right) / k_{\mathrm{B}} T}\right)^{N} .
$$

$k_{\mathrm{B}}$ and $T$ are the Boltzmann constant and absolute temperature, respectively. The case of isodesmic aggregation for onedimensional aggregates (rods) is worked out specifically by assigning a molecule-molecule "bond" energy of $\alpha k_{\mathrm{B}} T$ for each of the $(N-1)$ "bonds" in an aggregate of $N$ molecules,

$$
\mu_{N}^{0}=-\frac{N-1}{N} \alpha k_{\mathrm{B}} T .
$$

The volume fraction of molecules in aggregates of $N$ molecules is then

$$
X_{N}=N\left(X_{1} e^{\alpha}\right)^{N} e^{-\alpha} .
$$

If the total volume fraction of molecules in solution is $\phi$, then 


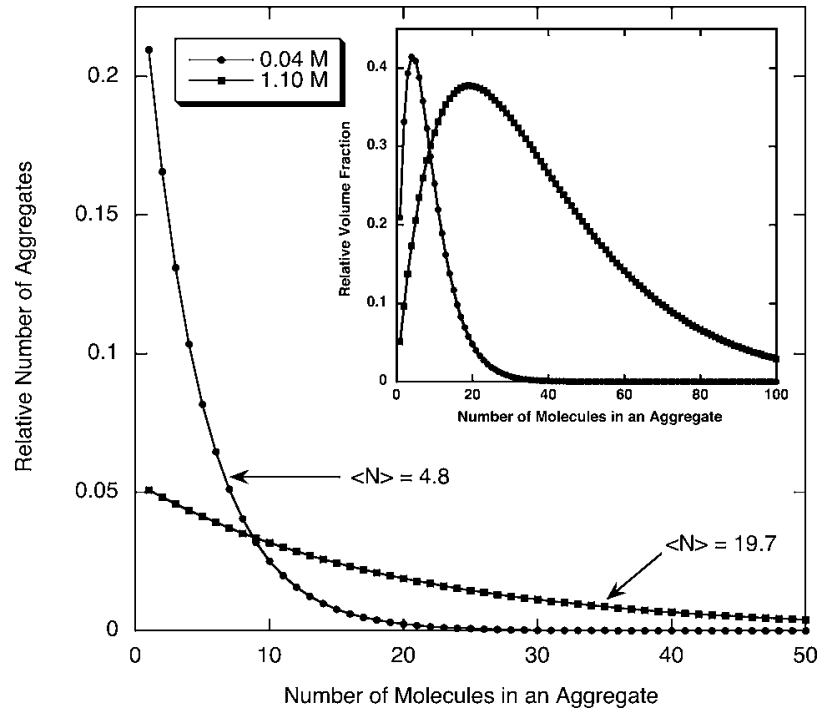

FIG. 1. Aggregate size distributions and volume fractions for two concentrations according to the simple theory using parameters appropriate to Sunset Yellow FCF at room temperature. The $0.04 M$ solution is far below the transition to the liquid crystal phase while the $1.10 \mathrm{M}$ solution represents a point on the phase diagram in the liquid crystal phase near the coexistence region. The numbers in angular brackets are the average number of molecules in an aggregate for each of the two distributions.

$$
\sum_{N=1}^{\infty} X_{N}=\sum_{i=1}^{\infty}\left[N\left(X_{1} e^{\alpha}\right)^{N} e^{-\alpha}\right]=\frac{X_{1}}{\left(1-X_{1} e^{\alpha}\right)^{2}}=\phi
$$

This equation can be solved for $X_{1}$,

$$
X_{1}=\frac{\left(1+2 \phi e^{\alpha}\right)-\sqrt{1+4 \phi e^{\alpha}}}{2 \phi e^{2 \alpha}}
$$

where it is clear that the distribution of aggregate sizes depends on the volume fraction $\phi$, the "bond" energy $\alpha$, and the temperature $T$.

These simple theoretical ideas for noninteracting aggregates should be most applicable in dilute systems and can easily be compared to experimental results if the density of the aggregated molecules $\rho$ (or the volume of a molecule in an aggregate) is known. The relationship between volume fraction and molar concentration $C_{\mathrm{M}}$ is

$$
C_{\mathrm{M}}=\frac{\rho \phi}{M(1-\phi)},
$$

where $\rho$ is the density in grams per $1000 \mathrm{~cm}^{3}$ and $M$ is the molar mass of the chromonic compound in grams per mole. As explained later, absorption and x-ray measurements allow the value of $\alpha$ to be determined for Sunset Yellow FCF. If this value is used, then the distribution of aggregate size can be calculated. Figure 1 contains the results of such a calculation for two concentrations, $0.04 M$ and $1.1 M$, at room temperature with $\rho=1.4 \mathrm{~g} / \mathrm{cm}^{3}[24]$ and $\alpha=7.25$. Quantities proportional to both $X_{N} / N$ and $X_{N}$ are plotted in Fig. 1. The former is proportional to the number of aggregates of each size, while the latter is the volume fraction of molecules in aggregates containing $N$ molecules. Notice that the $X_{N}$ distribution peaks while the $X_{N} / N$ distribution does not. $X_{N} / N$ becomes an exponential distribution in the limit of large $\phi e^{\alpha}$ [30]. The average aggregation number $\langle N\rangle$ is given for each concentration and is simply the average value of $N$ weighted according to the $X_{N} / N$ distribtuion. The more dilute of these two concentrations corresponds to a volume fraction of roughly 0.01 and is far below the transition to the liquid crystal phase. Notice that according to the theory there are on average only 4.8 molecules in an aggregate and there are few aggregates with over 10 molecules. The more concentrated solution has a volume fraction of approximately 0.26 which is just above the transition to the liquid crystal phase at room temperature in Sunset Yellow FCF. According to the theory, the average number of molecules in an aggregate is 19.7 and there is a significant number of aggregates containing more than 25 molecules. Assuming a repeat distance of $0.34 \mathrm{~nm}$ between the molecules and a molecular diameter of $1.2 \mathrm{~nm}$ (representative values for Sunset Yellow FCF), this implies a length-to-diameter ratio for the average aggregate of about 6 .

In comparing theory and experiment, a decision must be made concerning the two sodium atoms of Sunset Yellow FCF. In water, the sodium ions dissociate leaving the rest of the Sunset Yellow FCF molecule as a anion. The question then arises as to whether the sodium ions should be considered part of the aggregating molecule or part of the solution. The situation is made more complicated by the fact that a small association of the two oppositely charged ions is present, plus there is some association of water molecules with each Sunset Yellow FCF anion. Both of these effects make the chromonic molecule slightly larger than the Sunset Yellow FCF anion. In light of this, in all calculations the sodium atoms are considered to be part of the chromonic molecules that aggregate. This assumption has no qualitative effect on the results, because assuming the chromonic molecule is the Sunset Yellow FCF anion merely shifts the theoretical calculations along the concentration axis by $10 \%$.

More often than not, the absorption spectra of chromophores change when aggregation occurs. In some cases the change can be dramatic, as is the case for high concentrations of pseudoisocyanine chloride for which a strong, redshifted peak appears at a wavelength outside of the absorption region of isolated molecules [12-14]. For many chromophores at low concentrations, Blue 67 [22], disodium cromoglycate [11], Blue 27 [11], and Sunset Yellow FCF being examples, aggregation produces only modest changes in the strength and shape of the absorption band. Theoretical work in understanding these changes focuses on the electronic states of the molecule when coupling with nearby molecules in the aggregate is introduced. The general finding is that the absorption spectrum changes most when two molecules aggregate, and the change with the addition of more molecules gets less and less as the number of molecules in the aggregate increases.

One simple example of such a theoretical approach models the absorption as due to excitons. Coupling with other molecules is taken into account by introducing off-diagonal elements in the Hamiltonian for the aggregate [31]. If a constant amount of coupling is restricted to neighboring molecules, then the Hamiltonian for the system (dimension equal 
to the number of molecules in the aggregate) has the transition energy for the isolated molecule for each of the elements along the diagonal and a coupling constant for each element on either side of the diagonal. The energy eigenstates for such an aggregate can be found analytically and are given by

$$
E_{m}=E_{0}+2 \beta \cos \left(\frac{m \pi}{N+1}\right),
$$

where $m$ is the energy eigenstate index, $E_{0}$ is the transition energy of an isolated molecule, $N$ is the number of molecules in the aggregate, and $\beta$ is the coupling constant. According to this theory, the number of energy eigenstates increases linearly with $N$, but the separation between the highest and lowest energy eigenstates approaches an upper limit with increasing $N$.

In order to apply this theoretical model to Sunset Yellow FCF, in which the strength of the absorption decreases significantly with only a small shift in absorption wavelength, it is noted that the cosine function in Eq. (7) describes the decreasing nature of the changes to the absorption spectrum as molecules are added to larger and larger aggregates. It is reasonable to assume, therefore, that the decrease in absorption at a typical wavelength in the absorption region roughly follows such a functional form. If $a_{1}$ is the absorption coefficient at a specific wavelength for isolated molecules, then the absorption coefficient at that same wavelength for an aggregate of $N$ molecules $a_{N}$ can be expressed as follows:

$$
a_{N}=a_{1}+2 \beta \cos \left(\frac{\pi}{N+1}\right) \text {. }
$$

Using this functional form, the absorption coefficient changes from $a_{1}$ for isolated molecules to $a_{1}+2 \beta$ for infinitely long aggregates.

\section{EXPERIMENTAL PROCEDURES AND RESULTS}

Sunset Yellow FCF was purchased from Sigma-Aldrich and purified by dissolving it in millipore water, adding ethanol to cause the Sunset Yellow FCF to precipitate, filtering to isolate the precipitate, and then drying the powder in a vacuum oven. This procedure was performed twice before using the Sunset Yellow FCF in experiments. Solutions were prepared by mixing known amounts of purified Sunset Yellow FCF and millipore water, quickly sealing the container, and vortexing the mixture until the Sunset Yellow FCF dissolved completely. Solutions were pipetted into sample containers of various kinds, which were then sealed to prevent evaporation. Some of the absorption experiments and all of the liquid crystal experiments required thin samples, in which case a drop of solution was placed at the opening between two pieces of glass and the space between the two pieces of glass filled due to capillary action. This filling procedure was done in a humidity chamber to prevent evaporation during the 5-10 min it sometimes took for samples to fill. The capillary tubes used in the x-ray experiments were filled by placing a drop of solution in the reservoir end of the capillary tube and shaking the tube to force the solution into the thin portion of the capillary tube.

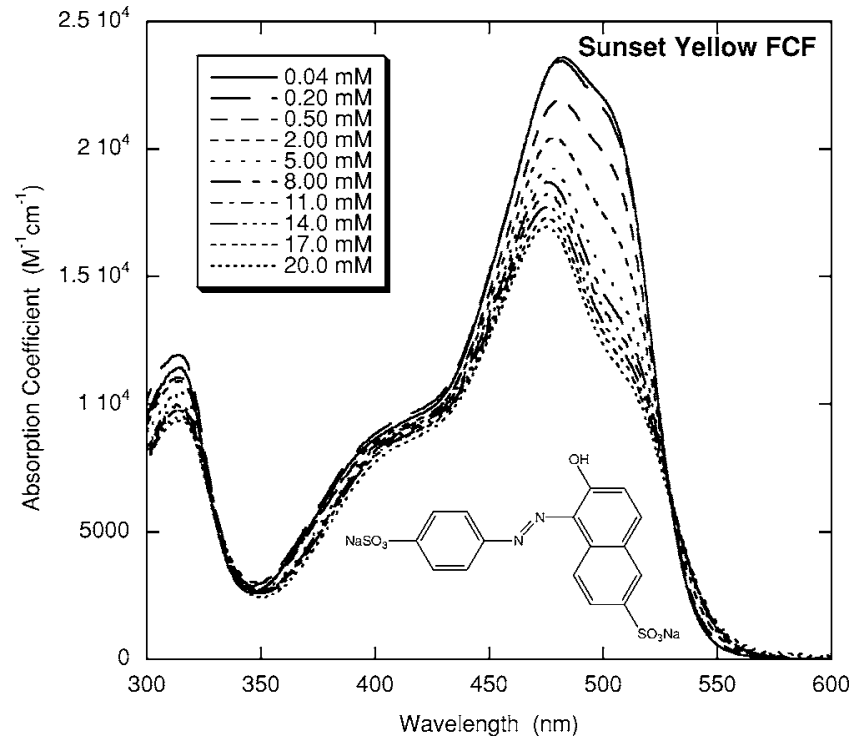

FIG. 2. Absorption coefficients in the uv-visible range for many dilute solutions of Sunset Yellow FCF (molecular structure also shown).

Absorption measurements were made using dilute solutions with concentrations ranging from $0.04 \mathrm{~m} M$ or $20 \mathrm{~m} M$. Use of sample containers with path lengths ranging from $50 \mu \mathrm{m}$ to $1 \mathrm{~cm}$ were necessary in order to keep the absorption within a useful range for the Jasco uv-visible spectrophotometer. The absorption spectra for all samples as well as the structure of Sunset Yellow FCF are shown in Fig. 2. Notice that while there is a significant change in the absorption coefficient as the concentration is increased, the shape of the absorption spectrum only changes a little. To check whether scattering might also be present, light scattering measurements were performed on solutions with concentrations up to the coexistence region. In all cases, the intensity of the scattered light was extremely weak, an indication that the size of the aggregates is significantly smaller than the wavelength of light.

The phase diagram for the Sunset Yellow FCF-water system was determined by making solutions with concentrations around $1 \mathrm{M}$, introducing these solutions into glass cells (10 $\mu \mathrm{m}$ path length), sealing the cells with epoxy and Critoseal®, placing the cells in a temperature controlled heating stage $\left(0.01{ }^{\circ} \mathrm{C}\right.$ precision $)$, and observing the cells through a polarizing microscope. With the polarizers of the microscope crossed, the isotropic phase appeared dark and the liquid crystal phase appeared bright yellow-orange. The temperature was increased and decreased at a rate of $0.4{ }^{\circ} \mathrm{C} / \mathrm{min}$ for each sample, recording the temperature at which one phase first appeared and then the temperature at which the other phase completely disappeared. The results of these measurements are plotted in Fig. 3, where the error bars represent the difference in temperatures recorded in heating and cooling. Notice that there is a significant coexistence region, which is not surprising given that there is a distribution of aggregate lengths. The phase diagram shown in Fig. 3 is very similar to the phase diagram contained in Ref. [24], with the location of the coexistence region differing by less than $0.1 \mathrm{M}$ and 


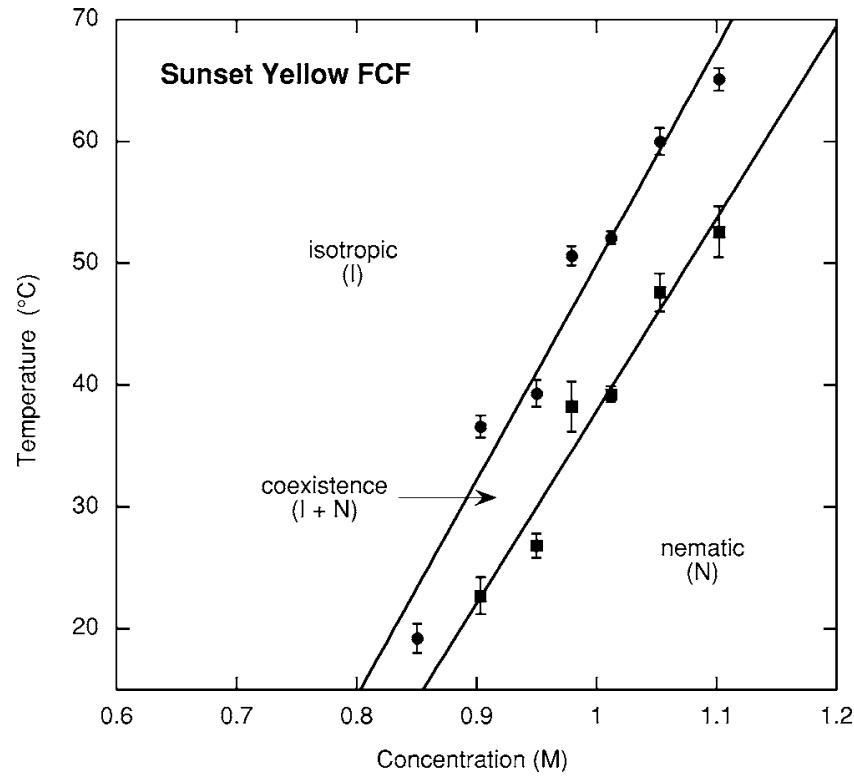

FIG. 3. Phase diagram for Sunset Yellow FCF. The solid circles and squares represent measurements of the isotropic-coexistence and nematic-coexistence boundaries, respectively. The error bars denote the difference between measurements taken on cooling and heating.

the slope of the coexistence region differing by a few percent.

$\mathrm{X}$-ray scattering experiments employed $\mathrm{Cu} K \alpha$ radiation from a Bruker-Nonius FR591 generator with mirrormonochromator optics and a multiwire detector. Samples were loaded into thin-walled capillary tubes, and for samples in the liquid crystal phase, there was evidence for some flow alignment of the director. Similar to the results for disodium cromoglycate [5] and in agreement with prior work on Sunset Yellow FCF [24], a broad concentration-dependent peak was observed at low angles, a sharper concentrationindependent peak occurred at high angles, and in partially aligned samples these peaks were separated by $90^{\circ}$ in $\chi$ scans. These results indicate that Sunset Yellow FCF is similar to disodium cromoglycate in that the molecules are stacked more or less normal to the columnar axis, giving a repeat distance along the axis of $0.34 \mathrm{~nm}$. In order to obtain more direct evidence for a structure, attention was concentrated on the smaller angle peak over a large concentration range. The results are displayed in Fig. 4, showing the shift of the x-ray peak with concentration. The horizontal axis denotes the scattering wave vector $q$,

$$
q=\frac{4 \pi}{\lambda} \sin \theta
$$

where $\lambda$ is the wavelength of the $\mathrm{x}$ rays and $\theta$ is the Bragg angle (half the scattering angle). The lines in the figure represent fits to the data of a Lorentzian line shape with a linear background.

An experiment on the highest concentration solution was also performed to investigate how the low angle peak depended on temperature. Data were obtained in the liquid

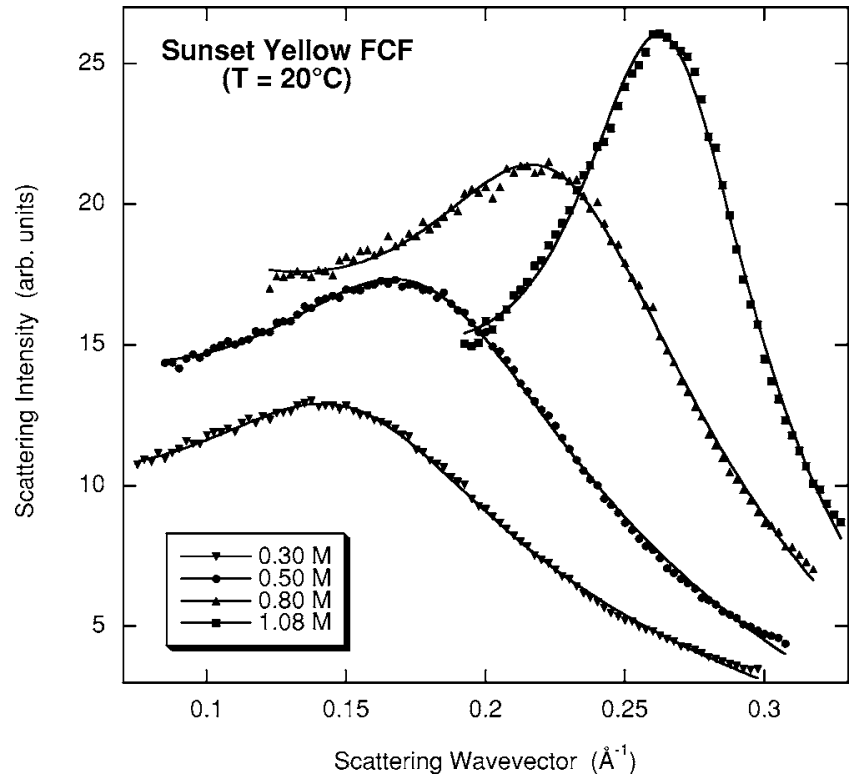

FIG. 4. X-ray scattering intensity as a function of scattering wave vector for three concentrations in the isotropic phase and one concentration in the nematic phase $(1.08 M)$. The lines are fits to the data of a Lorentzian function with a linear background.

crystal phase, across the coexistence region, and into the isotropic phase. As can be seen from Fig. 5, there is little change of the peak scattering wave vector in the nematic phase, with a small decrease inside the coexistence region. This indicates that the average separation between aggregates is approximately $2.5 \mathrm{~nm}$ and only weakly affected by the phase transition. On the other hand, as expected the full width at half maximum of the peak, which is inversely related to the distance over which motion of the aggregates is correlated, increases at all temperatures, especially across the nematic-isotropic transition. The correlation lengths obtained

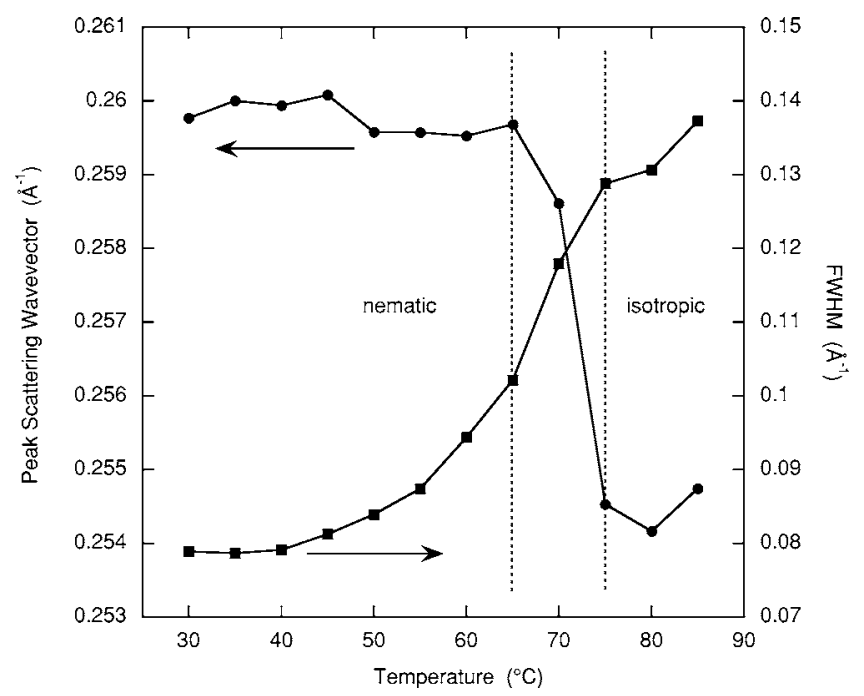

FIG. 5. Peak scattering wave vector and full width at half maximum of the x-ray scattering peak plotted against temperature for the $1.08 \mathrm{M}$ solution. The dotted lines indicate the location of the coexistence region between the nematic and isotropic phases. 
from the data range from about $8 \mathrm{~nm}$ at room temperature in the nematic phase to roughly $5 \mathrm{~nm}$ in the isotropic phase near the nematic-isotropic transition.

Measurements of the birefringence and order parameter of the liquid crystal phase require that the director be aligned. This can be difficult in a chromonic system, but it was achieved by allowing Sunset Yellow FCF in the liquid crystal phase to flow into the $10 \mu \mathrm{m}$ space between two pieces of glass. Rubbed glass and rubbed polyimide coated glass both induced alignment. Sometimes a small amount of suction was necessary to draw the liquid crystal into the opening. Sometimes the cell would slowly fill on its own in a humidity chamber. Because the alignment was never perfect, measurements of the birefringence and order parameter were performed using a microscope focused on a well aligned region of the sample (about $0.8 \mathrm{~mm}$ in diameter) as described in the following paragraphs.

Birefringence measurements were made by having linearly polarized light incident on the sample with its polarization at $45^{\circ}$ to the director. The light then passed through a quarter wave plate with its fast axis oriented parallel to the direction of incident light polarization. This resulted in linearly polarized light, but at an angle to the initial polarization direction equal to half the phase retardation introduced by the sample. The birefringence $\Delta n$ can then be found from the phase retardation $\psi$ by the following relation,

$$
\Delta n=\frac{2 \pi \lambda_{0} \psi}{d},
$$

where $\lambda_{0}$ is the wavelength of light in a vacuum and $d$ is the sample thickness. Since the measurement angle is the same for retardations that differ by $\pi$, the birefringence of a sample in a cell of half the thickness was also measured. Only one choice of the retardation angles for samples of both thicknesses yielded consistent values for the birefringence.

The results of these measurements are displayed in Fig. 6. Notice that the birefringence is negative in all cases, meaning that the index of refraction for light polarized parallel to the director is less than the index of refraction for light polarized perpendicular to the director. In a thermotropic liquid crystal composed of molecules with an $\mathrm{N}=\mathrm{N}$ bond and associated aromatic rings parallel to the long axis of the molecule, the birefringence is positive. If the $\mathrm{N}=\mathrm{N}$ bond in the Sunset Yellow FCF molecules lies perpendicular to the long axis of the aggregate, more $\mathrm{N}=\mathrm{N}$ bonds are perpendicular to the preferred direction of the aggregate long axis (director) than parallel to it. If the $\mathrm{N}=\mathrm{N}$ bond and associated aromatic rings dominate the optical response of this molecule, then the birefringence will be negative. Notice also that the magnitude of the birefringence increases as the concentration increases.

If just about all of the absorption of a molecule occurs for light parallel to one bond, the order parameter of that bond can be easily measured [32]. This is true for the $\mathrm{N}=\mathrm{N}$ bond of Sunset Yellow FCF just as long as the wavelength of light is in the absorption region. Order parameter measurements were made using the microscope and the same method of sample preparation. Parallel polarizers were placed on both sides of the sample, and the transmitted intensity was mea-

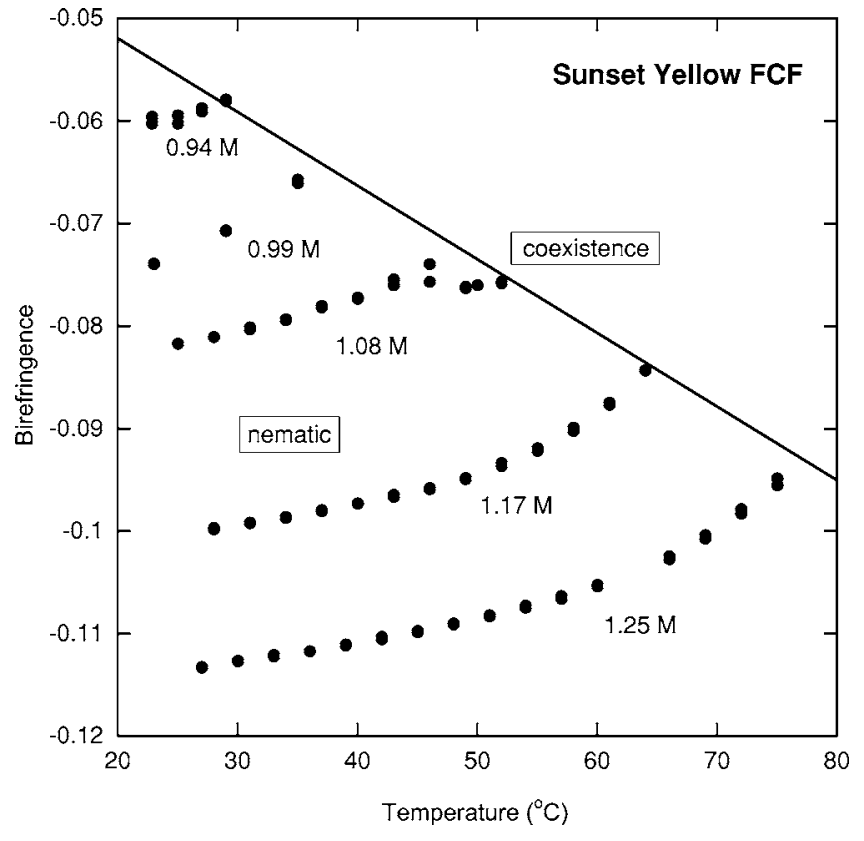

FIG. 6. The temperature dependence of birefringence for a number of concentrations. The wavelength of the light is $633 \mathrm{~nm}$. Since the nematic-isotropic temperature depends on concentration (see Fig. 3), the coexistence region occurs at different temperatures for each concentration.

sured with the director parallel and perpendicular to the polarizers. In order to obtain a reasonable amount of absorption in a $10 \mu \mathrm{m}$ thick sample, $576 \mathrm{~nm}$ light was used. The amount of absorption was calculated by comparing the transmitted intensity of the sample cell and an identical cell filled with water. In order to measure the order parameter, the indices of refraction for light polarized parallel and perpendicular to the director must be known. These were calculated by first measuring the index of refraction of solutions of Sunset Yellow FCF with different concentrations in the isotropic phase with an Abbé refractometer. This isotropic or average index of refraction was linear with concentration and was extrapolated to a concentration of $1.25 \mathrm{M}$, the concentration of the sample used for the order parameter measurements. The next step was to measure the birefringence of the sample as described previously. If the index of refraction in the isotropic phase is denoted by $n_{0}$ and the indices parallel and perpendicular to the director are denoted as $n_{\|}$and $n_{\perp}$, respectively, then the following approximate relations can be used to find the two indices,

$$
n_{\|} \approx n_{0}+\frac{2}{3} \Delta n, \quad n_{\perp} \approx n_{0}-\frac{1}{3} \Delta n .
$$

Knowing both the absorptions and indices of refraction for light parallel and perpendicular to the director, the order parameter $S$ can be found from the relationship,

$$
S=\frac{n_{\|} A_{\|}-n_{\perp} A_{\perp}}{n_{\|} A_{\|}+2 n_{\perp} A_{\perp}},
$$

where $A_{\|}$and $A_{\perp}$ are the absorptions for light polarized parallel and perpendicular to the director, respectively [32]. 


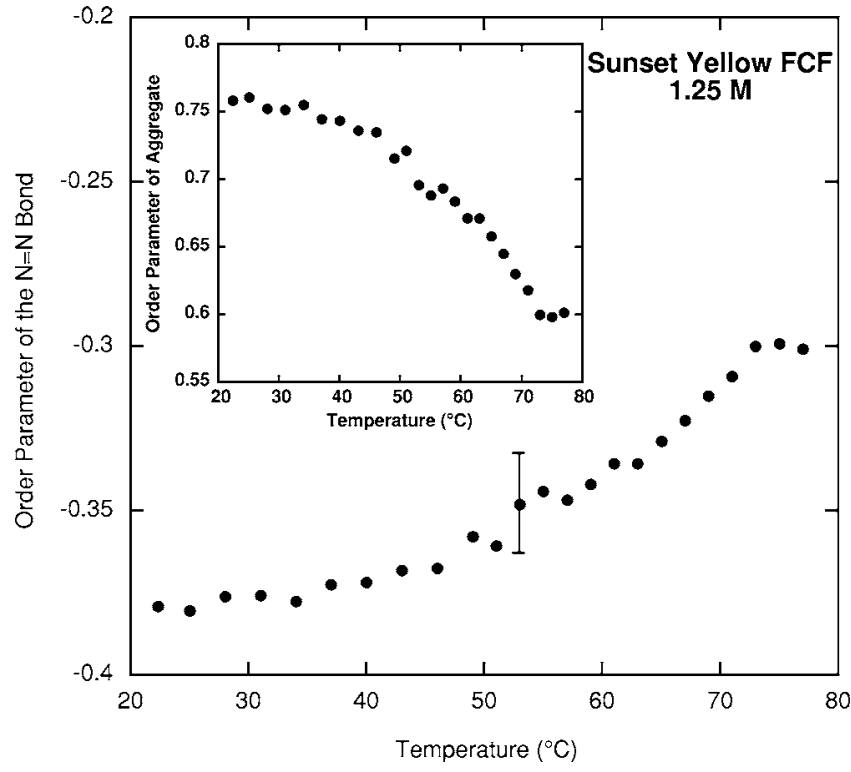

FIG. 7. Orientational order parameters as a function of temperature for a $1.25 \mathrm{M}$ solution. The full plot shows the measured order parameter of the $\mathrm{N}=\mathrm{N}$ bond. The insert displays the order parameter for the long axis of the aggregates assuming the $\mathrm{N}=\mathrm{N}$ bond makes an angle of $90^{\circ}$ with the long axis of the aggregate.

The results of these measurements on one solution of Sunset Yellow FCF are shown in Fig. 7. Again, the order parameter is negative indicating that the $\mathrm{N}=\mathrm{N}$ bond is more likely to be perpendicular to the director than parallel to the director, just as was inferred from the birefringence measurements.

\section{DISCUSSION}

To quantify the change in absorption during aggregation, the absorption coefficient at $500 \mathrm{~nm}$ is plotted as a function of concentration in Fig. 8, showing that the absorption coefficient strongly depends on concentration at low concentrations, with the effect increasing more slowly as the concentration increases. Also shown in the figure is a fit of the simple aggregation theory to the data. Since the density of Sunset Yellow FCF has been measured [24], the only parameters in the fit are the "bond" energy and the absorption coefficient for very large aggregates. The fit to the absorption data is excellent and yields values for "bond" energy at room temperature and absorption coefficient for large aggregates of $(7.25 \pm 0.01) k_{\mathrm{B}} T$ and $(9570 \pm 10) M^{-1} \mathrm{~cm}^{-1}$, respectively. This value for the "bond" energy between molecules in an aggregate of roughly seven times $k_{\mathrm{B}} T$ is an interesting result, since it indicates a fairly strong driving mechanism toward aggregation. But this must be the case since aggregation is significant at very low concentrations. These absorption measurements combined with the excellent theoretical fit are firm evidence of how the aggregation proceeds in Sunset Yellow FCF. Aggregation does not begin at some critical value of the concentration. Nor is there an optimum aggregate size as in the formation of micelles in amphiphilic systems. Rather, the aggregation is isodesmic in that it occurs at all concentra-

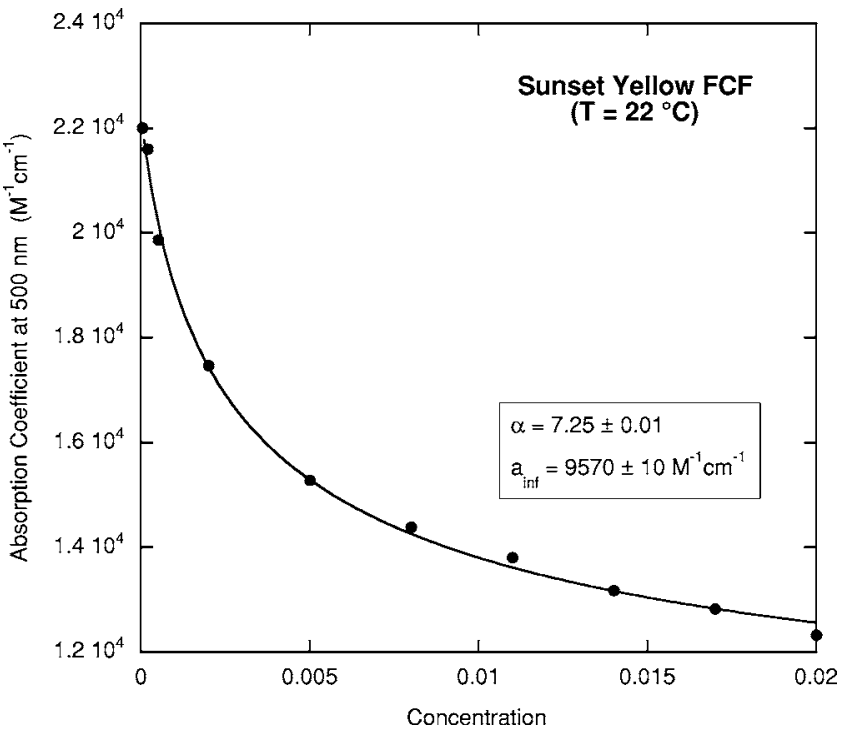

FIG. 8. Absorption coefficients for Sunset Yellow FCF at 500 $\mathrm{nm}$ as a function of concentration for dilute solutions. The line is a fit of the simple theory to the data as explained in the text.

tions, with the equilibrium distribution of aggregate sizes shifting to larger and larger aggregates as the concentration increases because the "bond" energy is independent of aggregate size. Thus at all concentrations there is a spread in the size of the aggregates, which is why a fairly large coexistence region is present. This type of isodesmic aggregation could very well describe chromonic liquid crystals in general.

The nematic liquid crystal phase of Sunset Yellow FCF forms at room temperature when the concentration reaches about $29 \mathrm{wt} \%$. This is a higher concentration than is typical for chromonic liquid crystals, which could be due to the fact that the cross section of the columns is one molecule. In hollow columns formed when there are several molecules in a cross-section, water is present in the center of the column, effectively reducing the amount of water between columns. In systems of water-filled columns, therefore, the nematic liquid crystal phase could very well occur at lower concentrations.

There is roughly a 7\% change in concentration from one boundary of the coexistence region to the other when the temperature is constant at $35^{\circ} \mathrm{C}$. This represents a change in the volume fraction of about $6 \%$ at an average volume fraction of 0.24 . According to the theory of Lee [26], a system with a length to diameter ratio of 11 has a similar volume fraction at the transition, which changes by $10 \%$ in going from one phase to another. With one set of parameters in the theory of Taylor and Herzfeld [27,28], a system with on average about 5 molecules in an aggregate has a similar volume fraction at the transition, which changes by $12 \%$ in crossing the coexistence region. On the other hand, this latter theory predicts that the width of the coexistence region for a fixed volume fraction of 0.24 is $13{ }^{\circ} \mathrm{C}$ if the transition takes place at $35^{\circ} \mathrm{C}$. This compares favorably with the experimental value of about $12{ }^{\circ} \mathrm{C}$. The slopes of the coexistence boundaries in temperature-volume fraction space in this same theory are 860 and $1040{ }^{\circ} \mathrm{C}$ for the nematic- 


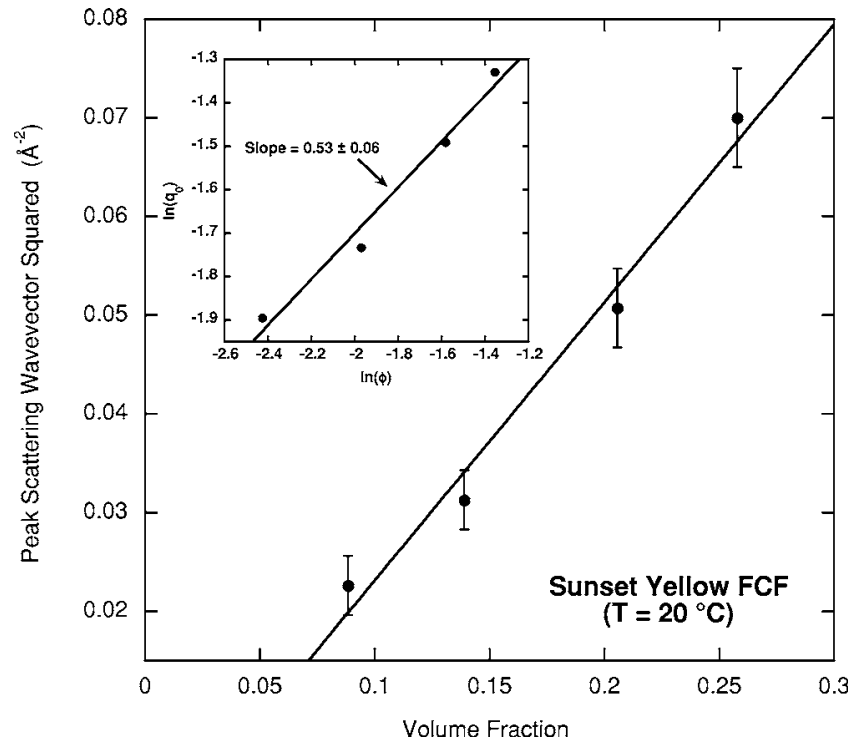

FIG. 9. Dependence of x-ray peak scattering wave vector on volume fraction. The inset is a log-log plot, while the full graph is a plot of the square of the x-ray peak scattering wave vector vs volume fraction. The lines are least square linear fits to the data.

coexistence and isotropic-coexistence boundaries, respectively. Experiment yields 840 and $917^{\circ} \mathrm{C}$ for the slopes of these boundaries.

One way to ascertain the shape of the aggregates is to investigate the dependence of the X-ray peak scattering wave vector on the volume fraction of the aggregates. This dependence is expected to be a power law, with the exponent revealing the number of dimensions into which the aggregates are packing. For example, if the x-ray peak scattering wave vector depends on volume fraction to the $1 / n$ power, $n$ indicates the number of packing dimensions. The inset in Fig. 9 shows a log-log plot of the $\mathrm{x}$-ray peak scattering wavevector vs. volume fraction for four solutions ranging from fairly dilute to a concentration in the liquid crystal phase. The slope of a linear fit to the data has a value of $0.53 \pm 0.06$, consistent with the packing of columns. The full plot in Fig. 9 displays a graph of the $\mathrm{X}$-ray peak scattering wavelength squared vs volume fraction, the slope of which reveals information on the cross-sectional area of the columns. For example, if hexagonal packing is assumed, the peak scattering wave vector $q_{0}$ is related to the volume fraction by

$$
q_{0}^{2}=\frac{2 \sqrt{3} \pi^{2}}{a} \phi,
$$

where $a$ is the cross-sectional area of the columns [33]. The linear fit shown in the figure yields a value for $a$ of $1.21 \pm 0.12 \mathrm{~nm}^{2}$, which is a perfectly reasonable value for the area of a single Sunset Yellow FCF molecule. If square packing of the columns is assumed, the value of $a$ increases by $13 \%$. Using a similar analysis but over a much narrower concentration range, Luoma estimates the diameter of the Sunset Yellow aggregates to be about $1.4 \mathrm{~nm}$ [24], a value indicating a slightly higher cross-sectional area.
The analysis of the X-ray measurements provides strong evidence that the structure of the aggregates in Sunset Yellow FCF is a columnar one, with single molecules in the stack. There is evidence that the aggregate structure in other chromonic systems may be different, although in most of these cases a firm conclusion is not yet possible.

The temperature dependence of the $\mathrm{x}$-ray scattering peaks is revealing. The average spacing between the aggregates decreases by approximately $2 \%$ at the transition from the isotropic to nematic phase. This represents a change in the density of the aggregates of roughly $0.04 \%$, which is an order of magnitude smaller than typical density changes at the nematic-isotropic transition in thermotropic liquid crystals [34]. This indicates the hard-rod character of the aggregates in this system, in which the density change at the transition results from a slight change in the distribution of aggregate sizes rather than a new balance between the intermolecular forces and entropy effects in a thermotropic system. The loss of orientational order at the transition is evident in the large change in the correlation length, which decreases by nearly a factor of 2 .

The birefringence data reveal a temperature dependence not unlike what is found in thermotropic liquid crystals. The absolute value of the birefringence is roughly proportional to the concentration and decreases slightly with a temperature increase until it starts to drop quickly to zero in the coexistence region. The fact that the birefringence is negative supports the model of the aggregates being columns with the molecules stacked in the columns so the $\mathrm{N}=\mathrm{N}$ bond is perpendicular to the long axis of the columns. Typical values for the indices of refraction of azo thermotropic liquid crystals with the $\mathrm{N}=\mathrm{N}$ bond parallel to the long molecular axis are $n_{\|}=1.8$ and $n_{\perp}=1.6$ [35]. However, the order parameter of thermotropic nematic liquid crystals is about half the value measured in Sunset Yellow FCF, and the concentration of a thermotropic liquid crystal is $100 \%$ as opposed to roughly $25 \%$ for Sunset Yellow FCF. Using Eq. (11), one might therefore estimate that the local indices of refraction parallel and perpendicular to the $\mathrm{N}=\mathrm{N}$ bond in Sunset Yellow FCF might be closer to 1.73 and 1.63 , respectively $\left(n_{0}\right.$ is assumed to remain the same since the final birefringence estimate does not depend on it). If 1.63 is used for the index of refraction for light polarized parallel to the column axis (perpendicular to the $\mathrm{N}=\mathrm{N}$ bond), and the average of 1.73 and 1.63 is used for the value of the index of refraction for light polarized perpendicular to the column axis, then a birefringence of about -0.05 is expected. This estimate is representative of the measured birefringence for the least concentrated solution. The birefringence of disodium cromoglycate, Blue 27, and Violet 20 has also been measured and falls within the range of values plotted in Fig. 6 [11].

The behavior of the order parameter for the $\mathrm{N}=\mathrm{N}$ bond shows a temperature dependence similar to what is typical in thermotropic liquid crystals. If there is no correlation between the orientational fluctuations of an aggregate relative to the director and the orientational fluctuations of the molecules within the aggregate relative to the aggregate axis, then there is a simple relationship between the order parameter of the aggregate long axis $S$ and the order parameter of the $\mathrm{N}=\mathrm{N}$ bond $S_{\mathrm{N}=\mathrm{N}}$, 


$$
S_{\mathrm{N}=\mathrm{N}}=\left\langle P_{2}(\cos \gamma)\right\rangle S,
$$

where $P_{2}$ is the second Legendre polynomial, the angular brackets denote a thermal average, and $\gamma$ is the angle between the $\mathrm{N}=\mathrm{N}$ bond and the long axis of the aggregate [36]. If $\gamma$ is assumed to be $90^{\circ}$, then the order parameter for the long axis of the aggregate can be calculated and is shown in the inset of Fig. 7. The order parameter of the long axes of the columns is between 0.6 and 0.75 , higher values than those found in thermotropic nematic liquid crystals, but this is expected for a system with some hard-rod character. Luoma determined the order parameter to be between 0.75 and 0.9 for Sunset Yellow FCF using x-ray diffraction, a method that may be more sensitive to larger aggregates [24]. Values found in disodium chromoglycate are around 0.75 [11]. Theoretically, the calculation for hard spherocylinders predicts an order parameter of 0.7 for a volume fraction corresponding to the Sunset Yellow FCF solution [26], while the result of the aggregation theory for this volume fraction gives an order parameter of 0.7 at the transition, increasing to 0.9 for the drop of temperature in Fig. $7[27,28]$.

\section{CONCLUSIONS}

This comprehensive investigation into the aggregation process and liquid crystal phase of a chromonic liquid crystal adds important information about a second chomonic system and confirms a general picture for the behavior of such systems. Sunset Yellow FCF shares many of the same properties as disodium cromoglycate, including an isodesmic aggregation process and the formation of columnar aggregates. The evidence pointing to columnar aggregates with a cross section of one molecule is strong in Sunset Yellow FCF. The birefringence and order parameter measurements represent some of the first measurements of this kind on these liquid crystal phases. Numerous possibilities exist for applications utilizing this aqueous liquid crystal phase with negative birefringence and intense absorption. A simple theory can explain the initial aggregation process, and there is reasonable agreement between the liquid crystalline data and a more complicated theory of aggregation.

\section{ACKNOWLEDGMENTS}

This work was supported by the National Science Foundation under Grant No. CHE-9900403 to Swarthmore College and Grant No. DMR-0102459 to the University of Pennsylvania, and by the Howard Hughes Medical Institute through a grant to Swarthmore College. Acknowledgment is made to the Donors of the American Chemical Society Petroleum Research Fund for partial support of this project. Fruitful discussions with S. Fraden, O. D. Lavrentovitch, R. B. Meyer, and R. F. Pasternack are gratefully acknowledged.
[1] J. Lydon, in Handbook of Liquid Crystals, edited by D. Demus, J. Goodby, G. W. Gray, H.-W. Spiess, and V. Vill (WileyVCH, New York, 1998), Vol. 2B, Chap. XVIII, p. 981.

[2] J. Lydon, Curr. Opin. Colloid Interface Sci. 3, 458 (1998).

[3] J. Lydon, Curr. Opin. Colloid Interface Sci. 8, 480 (2004).

[4] N. H. Hartshorne and G. D. Woodward, Mol. Cryst. Liq. Cryst. 23, 343 (1993).

[5] D. Goldfard, Z. Luz, N. Spielberg, and H. Zimmermann, Mol. Cryst. Liq. Cryst. 126, 225 (1985).

[6] Y. W. Hui and M. M. Labes, J. Phys. Chem. 90, 4064 (1986).

[7] Y. A. Nastishin, H. Liu, S. V. Shiyanovskii, O. D. Lavrentovich, A. F. Kostko, and M. A. Anisimov, Phys. Rev. E 70, 051706 (2004).

[8] J. E. Lydon, Mol. Cryst. Liq. Cryst. 64, 64 (1980).

[9] V. Ramesh, H.-S. Chien, and M. M. Labes, J. Phys. Chem. 91, 5937 (1987).

[10] S. V. Shiyanovskii, T. Schneider, I. I. Smalyukh, T. Ishikawa, G. D. Niehaus, K. J. Doane, C. J. Woolverton, and O. D. Lavrentovich, Phys. Rev. E 71, 020702(R) (2005).

[11] Y. A. Nastishin, H. Liu, T. Schneider, V. Nazarenko, R. Vasyuta, S. V. Shiyanovskii, and O. D. Lavrentovich, Phys. Rev. E 72, 041711(R) (2005).

[12] H. Stegemeyer and F. Stockel, Ber. Bunsenges. Phys. Chem. 100, 9 (1996).

[13] W. J. Harrison, D. L. Mateer, and G. J. T. Tiddy, J. Phys. Chem. 100, 2310 (1996).

[14] H. Rehage, G. Platz, B. Struller, and C. Thunig, Tenside, Surfactants, Deterg. 33, 242 (1996).
[15] D. Perahia, E. J. Wachtel, and Z. Luz, Liq. Cryst. 9, 479 (1991).

[16] V. A. Bykov, Y. G. Sharimanov, G. M. Mrevlishvili, T. D. Mdzinarashvili, N. O. Metreveli, and G. R. Kakabadze, Mol. Mater. 1, 73 (1992).

[17] G. J. T. Tiddy, D. L. Mateer, A. P. Ormerod, W. J. Harrison, and D. J. Edwards, Langmuir 11, 390 (1995).

[18] N. Usol'tseva, Mol. Cryst. Liq. Cryst. Sci. Technol., Sect. A 288, 201 (1996).

[19] C. Hahn, I. Spring, C. Thunig, G. Platz, and A. Wokaun, Langmuir 14, 6871 (1998).

[20] T. Schneider and O. D. Lavrentovich, Langmuir 16, 5227 (2000).

[21] K. Ichimura, T. Fujiwara, M. Momose, and D. Matsunaga, J. Mater. Chem. 12, 3380 (2002).

[22] C. Ruslim, D. Matsunaga, M. Hashimoto, T. Tamaki, andK. Ichimura, Langmuir 19, 3686 (2003).

[23] J. Turner, Ph.D. thesis, University of Leeds, 1988 (unpublished).

[24] R. J. Luoma, Ph.D. thesis, Brandeis University, 1995 (unpublished).

[25] L. Onsager, Ann. N.Y. Acad. Sci. 51, 627 (1947).

[26] S. D. Lee, J. Chem. Phys. 87, 4972 (1987).

[27] M. P. Taylor and J. Herzfeld, Langmuir 6, 911 (1990).

[28] M. P. Taylor and J. Herzfeld, Phys. Rev. A 43, 1892 (1991).

[29] P. K. Maiti, Y. Lansac, M. A. Glaser, and N. A. Clark, Liq. Cryst. 29, 619 (2002).

[30] J. Israelachvili, Intermolecular \& Surface Forces, 2nd ed. 
(Academic Press, London, 1992).

[31] J. Parkash, J. H. Robblee, J. Agnew, E. Gibbs, P. Collings, R. Pasternack, and J. C. de Paula, Biophys. J. 74, 2089 (1998).

[32] B. Badadur, Handbook of Liquid Crystals (Wiley-VCH, Weinheim, 1998), Vol. 2A.

[33] V. Luzzati, H. Mustacchi, A. Skoulios, and F. Husson, Acta Crystallogr. 13, 660 (1960).
[34] R. V. Tranfield and P. J. Collings, Phys. Rev. A 25, 2744 (1982).

[35] N. Tabiryan, U. Hrozhyk, and S. Serak, Phys. Rev. Lett. 93, 113901 (2004).

[36] P. J. Collings, S. I. Goss, and J. R. McColl, Phys. Rev. A 11, 684 (1975). 